DOI: https://doi.org/10.46296/yc.v5i9ucedespsoct.0119

\title{
EVALUACIÓN DEL PROGRAMA DE PRERREQUISITOS EN LA PRODUCCIÓN DEL QUESO GOUDA EN SIBANICÚ PARA EL DESARROLLO LOCAL
}

\section{EVALUATION OF THE PRERREQUISITOS PROGRAM IN THE GOUDA CHEESE PRODUCTION AT SIBANICÚ FOR THE LOCAL DEVELOPMENT}

\author{
Espinosa-Nieto Leticia ${ }^{\text {; }}$; Matos-Mosqueda Luisa ${ }^{2}$; León-Valle Wilson ${ }^{3}$; \\ Curbelo-Hernández Caridad ${ }^{4}$
}

1 Universidad de Camagüey "Ignacio Agramonte Loynaz". Camagüey, Cuba. Correo: leticia.espinosa@reduc.edu.cu. ORCID ID: https://orcid.org/0000-0002-4181-4276

${ }^{2}$ Universidad de Camagüey "Ignacio Agramonte Loynaz". Camagüey, Cuba. Correo: luisa.matos@reduc.edu.cu. ORCID ID: https://orcid.org/0000-0002-2387-163X

${ }^{3}$ Universidad Estatal Península de Santa Elena. Santa Elena, Ecuador. Correo: bleon@upse.edu.ec. ORCID ID: https://orcid.org/0000-0002-3374-5207

${ }^{4}$ Universidad Tecnológica de La Habana "José Antonio Echeverría". La Habana, Cuba. Correo: cary@quimica.cujae.edu.cu. ORCID ID: https://orcid.org/0000-0001-5191-7933

\section{Resumen}

Garantizar las condiciones higiénicas y sanitarias de los procesos de producción de alimentos constituye un compromiso de directivos y trabajadores, dada su incidencia en la inocuidad de los alimentos. El objetivo del presente trabajo es mostrar los resultados obtenidos en la evaluación del programa de prerrequisitos establecido en la producción del queso Gouda, así como las acciones propuestas para garantizar la condición higiénico-sanitaria del proceso, que permitan la implementación eficaz del Análisis de Peligros y Puntos Críticos de Control (APPCC). Para realizar la evaluación, se aplicó una lista de verificación basada en los Procedimientos Operacionales Estandarizados de Saneamiento establecidos en la fábrica, donde se obtuvo un porcentaje de cumplimiento de 59\%. Las principales deficiencias radican en las condiciones de la infraestructura, que traen consigo impedimentos para llevar a cabo correctamente la limpieza y desinfección. También se producen violaciones de lo establecido en los documentos normativos, lo que conlleva a la falta de higiene del personal y de seguridad del proceso. Se identifican como causas principales la insuficiente gestión de recursos, la falta de responsabilidad y las deficiencias del diseño y montaje, para cuya solución se elabora una propuesta de acciones, con vistas a garantizar las condiciones higiénico-sanitarias del proceso de producción para mejorar la imagen de la instalación.

Palabras claves: programa de prerrequisitos; Análisis de Peligros y Puntos Críticos de Control; condiciones higiénico-sanitarias.

\begin{abstract}
Ensuring the hygienic and sanitary conditions of food production processes is a commitment of manegers and workers, given their impact on food safety. The objective of this work is to show the results obtained in the evaluation of the prerequisite program established in the production of Gouda cheese, as well as the actions proposed to guarantee the hygienic-sanitary condition of the process, which will allow the effective implementation of the Hazard Analysis and Critical Control Points (HACCP). For the purpose of carrying out the assessment, a checklist based on the Standardised Operational Sanitation Procedures established at the plant, was applied, where a compliance rate of $59 \%$ was obtained. The main deficiencies lie in the infrastructure conditions,
\end{abstract}

Información del manuscrito:

Fecha de recepción: 26 de julio de 2021.

Fecha de aceptación: 29 de septiembre de 2021.

Fecha de publicación: 11 de octubre de 2021. 
which lead to impediments to proper cleaning and disinfection. There are also violations of the provisions of the normative documents, which leads to a lack of hygiene for the staff and the safety of the process. The main causes are the insufficient management of resources, lack of responsibility and deficiencies in the design and assembly, for which a proposal for actions is prepared, with a view to guaranteeing the hygienic-health conditions of the production process to improve the image of the installation.

Keywords: Prerequisite program; Hazard Analysis and Critical Control Points; hygienic-sanitary conditions.

\section{Introducción}

La alimentación constituye una de las actividades esenciales de los seres humanos, pues responde a la necesidad fisiológica de incorporar nutrientes y energía que le permiten al organismo funcionar correctamente. Sin embargo, es considerada también una actividad de riesgo, porque los alimentos pueden ser portadores de contaminantes que causan Enfermedades Transmitidas por los Alimentos (ETAS) (Garayoa et al., 2010).

Para evitar las consecuencias perjudiciales que derivan de las enfermedades y los daños que provocan los alimentos contaminados para la salud y la economía, es imprescindible garantizar la inocuidad de los alimentos. Ésta ofrece seguridad de que el alimento no causará daños al consumidor cuando sea preparado e ingerido, por lo que se ha convertido en un parámetro necesario que promueve y proporciona confiabilidad en los consumidores y garantía para los productores, elaboradores y comercializadores (Díez, 2018).

En una Empresa de alimentos, La inocuidad es un aspecto fundamental del proceso de calidad que se tenga establecido, así como un asunto esencial de salud pública que involucra a los consumidores, productores y gobierno (Serrano, 2017) p 6. La inocuidad alimentaria se obtiene como resultado del aseguramiento de la calidad higiénico- sanitaria en el proceso de producción y elaboración de los productos alimenticios. Para ello se requiere la aplicación de un conjunto de medidas enfocadas en la prevención de la contaminación de los alimentos, en todas las etapas del ciclo producción. 
Es indispensable entonces, referirse al Análisis de Peligros y Puntos Críticos de Control (APPCC), las Buenas Prácticas de Manufactura (BPM) y los Procedimientos Operacionales Estándar de Saneamiento (POES), que, de conjunto, son capaces de diagnosticar, prevenir errores higiénico- sanitarios y de elaboración para así garantizar la protección sanitaria de los alimentos hasta alcanzar la inocuidad de los mismos (Ballesteros, 2021), pp 19-20.

El APPCC, se reconoce internacionalmente como el mejor método para garantizar la seguridad de los productos y para controlar los riesgos originados por los alimentos. Constituye una herramienta eficaz, dirigida a la identificación, evaluación y control de los peligros asociados con las materias primas, ingredientes, procesos, ambiente, comercialización y su uso por el consumidor, a fin de garantizar la inocuidad del alimento. Este sistema tiene fundamentos científicos, un carácter sistemático y está caracterizado por un enfoque preventivo de los riesgos sanitarios vinculados a los alimentos. Es decir, está enfocado hacia el control de las etapas críticas para la inocuidad del alimento a diferencia del control tradicional que se basa en la inspección de las instalaciones y el análisis del producto final (Cunalata y Gabriela, 2017).

Sin embargo, para la implementación de un sistema APPCC, es imprescindible contar con las condiciones higiénicas y sanitarias del proceso. Esto se logra mediante el establecimiento de programas de prerrequisitos, los cuales son usados para el control de las condiciones de la planta y el ambiente; y representan la suma de los programas, prácticas y procedimientos que deben ser aplicados para diseñar, producir y distribuir alimentos inocuos (Otegui, Michelini y Civit, 2017).

Los programas de prerrequisitos deben aplicarse antes y durante la implantación del Sistema APPCC ya que son esenciales para mantener un ambiente higiénico apropiado para la producción, manipulación y provisión de productos finales para el consumo humano. Se refieren al control de aspectos que pueden suponer un peligro y afectar a la seguridad alimentaria en todas 0 al menos varias de las etapas del 
proceso productivo. Es la herramienta crucial para que el sistema APPCC funcione eficazmente (Palomino, 2018).

Los programas de prerrequisitos están conformados por los POES, los cuales comprenden los procederes que describen las tareas de limpieza y desinfección destinadas a mantener o restablecer las condiciones de higiene del local, equipos y procesos de elaboración de alimentos para prevenir la aparición de ETAs. También forman parte de los programas de prerrequisitos, las BPM, éstas consideran los principios básicos y las prácticas generales de higiene durante la manipulación, preparación, elaboración, envasado, almacenamiento, transporte y distribución de alimentos para consumo humano, a fin de que se disminuyan los riesgos inherentes a la producción (Campoverde, 2015).

La implementación de sistemas APPCC y el cumplimiento de los programas de prerrequisitos, es de gran importancia para la industria quesera. El queso es un producto potencialmente peligroso ya que puede verse afectado por diversas fuentes de contaminación como microorganismos $y$ ha estado implicado como vehículo de enfermedades transmitidas por los alimentos (Vargas, 2016).

En la fábrica de quesos del municipio Sibanicú, provincia de Camagüey, se produce el queso Gouda. Esta variedad de queso se comercializa en todo el país, principalmente en la zona oriental. Sin embargo, se pretende que la mayor parte de las producciones se destinen al sector turístico, lo cual requiere la demostración de que se asegura la inocuidad de este producto. A pesar de que la fábrica tiene diseñado un programa de prerrequisitos, en inspecciones realizadas se ha confirmado que éste no garantiza que se cumplan correctamente los requisitos higiénicos-sanitarios del proceso, los cuales constituyen el elemento básico para lograr el funcionamiento eficaz del sistema HACCP. En este sentido, fue necesario llevar a cabo una investigación, con la finalidad de realizar una evaluación del programa de prerrequisitos existente, para determinar los factores limitantes de su correcta implementación. El presente trabajo tiene como objetivo mostrar los resultados de la 
evaluación del programa de prerrequisitos realizada en la fábrica de quesos del municipio Sibanicú y las acciones propuestas para la mejora de las condiciones higiénicosanitarias del proceso de producción del queso Gouda.

\section{Metodología}

La presente investigación se llevó a cabo en la fábrica de quesos Sibanicú, durante un período de tres meses. Para su desarrollo se utilizó un procedimiento metodológico de elaboración propia, tomando como referencia procederes descritos en la literatura consultada: (Castañeda, Fuentes y Peñarrieta, 2016); (Cachay y Velezmoro, 2019); (Saltos, Márquez, Demera, Alcívar, 2019).

El procedimiento metodológico utilizado consta de cinco etapas, las cuales fueron diseñadas de acuerdo con los requerimientos del proceso investigado.

I. Preparación del proceso de evaluación de los prerrequisitos.

II. Recopilación de datos.
III. Procesamiento estadístico de los datos recopilados.

IV. Análisis de los resultados obtenidos.

V. Propuesta de acciones para la mejora del cumplimiento del programa de prerrequisitos.

Etapa I. Preparación del proceso de evaluación de los prerrequisitos.

El programa de prerrequisitos diseñado para la producción de queso Gouda en la fábrica de Sibanicú, está conformado por ocho Procedimientos de Operación Estandarizados de Saneamiento (POES), éstos son: limpieza y desinfección; infraestructura y mantenimiento; control del agua; control de plagas y vectores; control de productos químicos; gestión de residuos sólidos y líquidos; control de materias primas; higiene del personal y capacitación. En la primera etapa se desarrollaron tres actividades, las cuales se relacionan a continuación.

a) Se comprobó la correspondencia de los POES con las Normas Cubanas que establecen los requisitos de obligatorio cumplimiento. Al 
realizar la comparación, se pudo comprobar que existía correspondencia entre éstos, por lo que los POES fueron utilizados como referente para confeccionar las herramientas de recopilación de datos y definir los criterios de evaluación.

b) Se identificaron los parámetros a utilizar en la evaluación, así como los criterios de comparación, según los POES establecidos. Como resultado se definieron ocho variables, conformadas, en total, por 156 parámetros. En dependencia de la naturaleza de cada uno de los parámetros a evaluar, se decidió el método a utilizar para la recogida de datos

c) Se elaboraron las herramientas para la recogida de datos. Fueron diseñados cuestionarios de preguntas, listas de verificación, guías de observación y encuestas. Estas herramientas abarcaron todos los parámetros a evaluar en ocho variables.
Etapa II. Recopilación de datos.

El proceso de recopilación de datos se llevó a cabo durante diez semanas de producción. Este proceso se desarrolló mediante la utilización de métodos como la observación, revisión de documentos y entrevistas, con el apoyo de las herramientas de recogida de datos diseñadas.

Etapa III. Procesamiento estadístico de los datos recopilados

El volumen de datos obtenidos fue procesado estadísticamente, lo cual permitió la determinación de la cantidad de requisitos cumplidos e incumplidos, así como sus respectivos porcentajes, estratificados por variables y de manera general.

Otro elemento que se tuvo en cuenta, fue la identificación de los principales problemas por variables, así como las causas asociados a cada uno de ellos.

Etapa IV. Análisis de los resultados obtenidos.

Con la información obtenida, se procedió a la valoración de la situación existente. Para calificar el 
nivel de cumplimiento del programa de prerrequisitos y de cada una de las variables que lo conforman se utilizó la siguiente escala:

De $90 \%$ - $100 \%$ de cumplimiento. Muy bien

De $70 \%-89 \%$ de cumplimiento. Bien

De $50 \%-69 \%$ de cumplimiento. Regular

De $30 \%-49 \%$ de cumplimiento. Mal

Menos de $30 \%$ de cumplimiento. Muy mal

A partir de los problemas identificados y sus respectivas causas, se procedió a la detección de aquellas causas de mayor incidencia en los incumplimientos de prerrequisitos.
Etapa V. Propuesta de acciones para la mejora del cumplimiento del programa de prerrequisitos.

Las causas que fueron identificadas como las de mayor incidencia en los incumplimientos de prerrequisitos, sirvieron de base para la elaboración de una propuesta de las acciones a desarrollar y un listado de los recursos necesarios, para su consideración en los planes de inversiones futuras.

\section{Resultados}

Los resultados obtenidos en la evaluación de cada una de las variables definidas para la evaluación de los prerrequisitos se presentan de manera resumida en la tabla 1, la cual se muestra a continuación:

Tabla 1. Resultados de la evaluación de los prerrequisitos.

\begin{tabular}{|l|c|c|c|c|c|}
\hline \multicolumn{1}{|c|}{ Variable } & $\begin{array}{c}\text { Total de } \\
\text { aspectos }\end{array}$ & Cumple & $\begin{array}{c}\% \\
\text { Cumple }\end{array}$ & Incumple & $\begin{array}{c}\% \\
\text { Incumple }\end{array}$ \\
\hline $\begin{array}{l}\text { Limpieza y } \\
\text { desinfección }\end{array}$ & 32 & 13 & 40,62 & 19 & 59,38 \\
\hline $\begin{array}{l}\text { Infraestructura y } \\
\text { mantenimiento }\end{array}$ & 37 & 18 & 48,65 & 19 & 51,35 \\
\hline Control de agua & 13 & 11 & 84,6 & 2 & 15,4 \\
\hline $\begin{array}{l}\text { Control de plagas y } \\
\text { vectores }\end{array}$ & 16 & 10 & 66,7 & 6 & 33,3 \\
\hline $\begin{array}{l}\text { Control de } \\
\text { productos químicos }\end{array}$ & 7 & 7 & 100 & 0 & 0 \\
\hline $\begin{array}{l}\text { Gestión de residuos } \\
\text { sólidos y líquidos }\end{array}$ & 14 & 9 & 64,3 & 5 & 35,7 \\
\hline
\end{tabular}




\begin{tabular}{|l|c|c|c|c|c|}
\hline $\begin{array}{l}\text { Control de materias } \\
\text { primas }\end{array}$ & 12 & 9 & 75 & 3 & 15 \\
\hline $\begin{array}{l}\text { Higiene } \\
\text { personal del } \\
\text { capacitación }\end{array}$ & 25 & 15 & 60 & 10 & 40 \\
\hline Total & $\mathbf{1 5 6}$ & $\mathbf{9 2}$ & $\mathbf{5 9}$ & $\mathbf{6 4}$ & $\mathbf{4 1}$ \\
\hline
\end{tabular}

- Análisis del nivel de cumplimiento de los prerrequisitos

De los 156 aspectos que conforman el programa de prerrequisitos evaluado, el $59 \%$ se cumplen, mientras que el $41 \%$ se incumplen total o parcialmente. Atendiendo a la escala utilizada para la calificación, puede decirse que el programa de prerrequisitos de la producción de queso gouda en la fábrica de Sibanicú, tiene un desempeño Regular. Respecto a las variables, el control de productos químicos califica como Muy Bien; el control de agua y control de materias primas, califican como Bien; el control de plagas y vectores, la gestión de residuos sólidos y líquidos y la higiene del personal y capacitación, obtienen la calificación de Regular; mientras que la limpieza y desinfección y la infraestructura y mantenimiento, tienen un comportamiento deficiente, por lo que califican como Mal.
- Análisis del comportamiento por variables

Al desagregar el análisis por variables se obtienen los siguientes resultados:

\section{Variable: Limpieza y desinfección}

Del total de 32 aspectos evaluados en relación con la variable limpieza y desinfección, se presenta un 59,38 $\%$ de incumplimientos. Dentro de los principales problemas detectados se encuentra la violación de lo estipulado en el plan de limpieza y desinfección de los equipos de trabajo y el área de proceso; poca sistematicidad de vigilancia por un especialista de calidad a la hora de la limpieza y desinfección $(\mathrm{L}+\mathrm{D})$ y no se usan todos los recursos necesarios. Estos problemas obedecen a las siguientes causas: falta de responsabilidad, insuficiente gestión de recursos, falta de mantenimiento, deficiencias en el diseño y montaje, así como capacitación insuficiente. 


\section{Variable: Infraestructura $y$ mantenimiento}

De los 37 aspectos evaluados el $51,35 \%$ se incumplen total o parcialmente. Los problemas que mayor incidencia tienen en este resultado son: el incumplimiento del plan de mantenimiento previsto, así como la falta de condiciones en los locales para garantizar el cumplimiento de las medidas higiénicas y sanitarias establecidas. Estos problemas responden, en gran medida, a insuficiente gestión de recursos y a las deficiencias en el diseño y montaje. Sin embargo, también inciden la falta de responsabilidad, la falta de mantenimiento y la falta de presupuesto.

\section{Variable: Control del agua}

De los 13 aspectos analizados el $15,39 \%$ se incumplen. Las principales deficiencias detectadas están relacionadas con la falta de sistematicidad en la cloración del agua y las dificultades para su control microbiológico, lo cual obedece a problemas financieros, dados por el presupuesto insuficiente.

\section{Variable: Control de plagas $y$ vectores}

En esta variable se evalúan 16 aspectos, de los cuales se incumple el $33,3 \%$. Los problemas que más inciden en los incumplimientos se corresponden con la falta de condiciones para impedir la entrada y proliferación de insectos y roedores a las instalaciones. Estos problemas tienen como causas fundamentales: la insuficiente gestión de recursos, la falta de responsabilidad y las deficiencias en el diseño y montaje.

\section{Variable: Control de productos químicos \\ Los siete aspectos analizados se cumplen satisfactoriamente.}

\section{Variable: Gestión de residuos sólidos y líquidos}

La variable gestión de residuos sólidos y líquidos reporta un 35,7\% de deficiencias con respecto a los 14 aspectos evaluados. Los problemas detectados responden incumplimientos de las regulaciones establecidas para la extracción y las condiciones requeridas para la disposición de los residuos. Ello obedece a la insuficiente gestión de 
recursos y a aspectos relacionados con el mal diseño de la instalación.

\section{Variable: Control de materias primas}

De los 12 aspectos analizados, se incumple el $25 \%$ Los incumplimientos están dados fundamentalmente por la falta de sistematicidad en la exigencia de las certificaciones de conformidad de las materias primas, lo cual obedece a la falta de responsabilidad.

Variable: Higiene de personal y capacitación

De los 25 aspectos evaluados, el 40 $\%$ se incumple total o parcialmente. Los problemas que mayor incidencia tienen en este resultado son las deficiencias en el cumplimiento de las medidas higiénicas y sanitarias establecidas, fundamentalmente las relacionadas con el incorrecto lavado de las manos, el uso incorrecto de la ropa sanitaria, el uso inadecuado de prendas y la ingestión de alimentos en áreas del proceso. Estos problemas responden a problemas de responsabilidad, así como a insuficiente gestión de recursos y a deficiencias en el diseño y montaje.

\section{- Relación entre los problemas}

\section{detectados y las causas asociadas}

Teniendo en cuenta que las causas identificadas resultan coincidentes para las diferentes variables, se realiza un análisis de la frecuencia de los problemas que tienen causas comunes, para la determinación de su relación. Los resultados del análisis se muestran en la Tabla 2.

Tabla 2. Cantidad de problemas detectados con causas comunes por variable.

\begin{tabular}{|c|c|c|}
\hline Variable & Causas identificadas & $\begin{array}{c}\text { Cantidad de problemas } \\
\text { por causa }\end{array}$ \\
\hline $\begin{array}{ll}\text { Limpieza } & y \\
\text { desinfección }\end{array}$ & $\begin{array}{l}\text { Deficiencias en diseño y montaje } \\
\text { Insuficiente gestión de recursos } \\
\text { Falta de responsabilidad } \\
\text { Falta de mantenimiento } \\
\text { Insuficiente capacitación }\end{array}$ & $\begin{array}{l}3 \\
5 \\
5 \\
3 \\
3\end{array}$ \\
\hline $\begin{array}{l}\text { Infraestructura y } \\
\text { mantenimiento }\end{array}$ & $\begin{array}{l}\text { Deficiencias en diseño y montaje } \\
\text { Insuficiente gestión de recursos } \\
\text { Falta de responsabilidad } \\
\text { Falta de mantenimiento } \\
\text { Presupuesto insuficiente }\end{array}$ & $\begin{array}{l}5 \\
7 \\
3 \\
2 \\
2\end{array}$ \\
\hline Control de agua & Presupuesto insuficiente & 2 \\
\hline $\begin{array}{l}\text { Control de plagas } \\
\text { y vectores }\end{array}$ & $\begin{array}{l}\text { Deficiencias en diseño y montaje } \\
\text { Insuficiente gestión de recursos }\end{array}$ & $\begin{array}{l}1 \\
4\end{array}$ \\
\hline
\end{tabular}




\begin{tabular}{|l|l|c|}
\hline & Falta de responsabilidad & 1 \\
\hline $\begin{array}{l}\text { Gestión de } \\
\text { residuos sólidos y } \\
\text { líquidos }\end{array}$ & $\begin{array}{l}\text { Deficiencias en diseño y montaje } \\
\text { Insuficiente gestión de recursos }\end{array}$ & 3 \\
\hline $\begin{array}{l}\text { Control de } \\
\text { materias primas }\end{array}$ & Falta de responsabilidad & 3 \\
\hline $\begin{array}{l}\text { Higiene del } \\
\text { personal } \\
\text { capacitación yalta de responsabilidad } \\
\text { Deficiencias en diseño y montaje } \\
\text { Insuficiente gestión de recursos }\end{array}$ & 3 \\
\hline
\end{tabular}

La frecuencia de problemas asociados a cada una de las causas identificadas se resume en la Tabla 3, la cual se muestra a continuación.

Tabla 3. Frecuencia de problemas asociados a cada causa.

\begin{tabular}{|l|c|}
\hline \multicolumn{1}{|c|}{ Causa } & Frecuencia \\
\hline Falta de responsabilidad & 15 \\
\hline Insuficiente gestión de recursos & 23 \\
\hline Presupuesto insuficiente & 4 \\
\hline Deficiencias en el diseño y montaje & 14 \\
\hline Falta de mantenimiento & 5 \\
\hline Insuficiente capacitación & 3 \\
\hline
\end{tabular}

Los datos anteriores ilustran que las causas que han tenido mayor incidencia en el incumplimiento del programa de prerrequisitos son la insuficiente gestión de recursos, la falta de responsabilidad y las deficiencias en el diseño y montaje, por lo que se le da prioridad a su tratamiento, mediante la propuesta de acciones que posibiliten su solución, las cuales se muestran en la Tabla 4.

Tabla 4. Propuesta de acciones.

\begin{tabular}{|c|c|}
\hline $\begin{array}{c}\text { Deficiencias a } \\
\text { solucionar }\end{array}$ & Propuesta de acciones \\
\hline $\begin{array}{l}\text { Insuficiente gestión de } \\
\text { recursos }\end{array}$ & $\begin{array}{l}\text { - Mayor exigencia por parte de los directivos hacia las } \\
\text { personas encargadas y responsables de realizar las } \\
\text { actividades de compra del establecimiento. } \\
\text { - Lograr la idoneidad del personal que realiza la } \\
\text { actividad de compra. } \\
\text { Elaborar proyectos que aporten financiamiento para la } \\
\text { adquisición de equipos y utensilios necesarios para el } \\
\text { proceso. }\end{array}$ \\
\hline
\end{tabular}




\begin{tabular}{|c|ll|}
\hline \multirow{2}{*}{ Falta de responsabilidad } & - $\begin{array}{l}\text { Establecer por escrito las funciones y } \\
\text { responsabilidades de cada trabajador. }\end{array}$ \\
& - $\begin{array}{l}\text { Capacitar y concientizar a cada trabajador en relación } \\
\text { con sus funciones y responsabilidades. }\end{array}$ \\
\hline - & Mayor exigencia por parte de los directivos. \\
\hline Deficiencias en el diseño & - Rediseñar los elementos del proceso que no estén \\
y montaje & acordes con los principios de la inocuidad alimentaria. \\
& $\begin{array}{l}\text { Elaborar proyectos para el rediseño, que aporten } \\
\text { financiamiento para llevar a cabo las inversiones } \\
\text { necesarias. }\end{array}$ \\
\hline
\end{tabular}

\section{Conclusiones}

La evaluación de los prerrequisitos permite la identificación de los aspectos que impiden que se garanticen las condiciones higiénicas y sanitarias en los procesos de producción de alimentos, por lo que constituye un paso esencial para llevar a cabo sistemas de Análisis de Peligros y Puntos Críticos de Control.

Mediante la aplicación del procedimiento metodológico propuesto, se constató que el desempeño del programa de prerrequisitos en el proceso de producción del queso Gouda es Regular, evidenciándose el incumplimiento del $41 \%$ de los aspectos evaluados, cuyas causas fundamentales radican en la insuficiente gestión de recursos, la falta de responsabilidad y las deficiencias en el diseño y montaje.
Las acciones propuestas pueden contribuir al cumplimiento de aspectos esenciales del programa de prerrequisitos, sin los cuales no es posible llevar a cabo un sistema de APPCC.

\section{Bibliografía}

Ballesteros, A. (2021) Impacto de la implementación del sistema de gestión de inocuidad alimentaria bajo la norma ISO 22000 en PYMES de la industria de alimentos. Monografía para optar al título de Especialista en Gerencia de la Calidad, Fundación Universidad de América, Bogotá, Colombia. Recuperado de: https://repository.uamerica.ed u.co

Cachay, N., Velezmoro, W. (2019). Diseño de las Buenas Prácticas de Manufactura (BPM) Y LOS Procedimientos Estándares de Saneamiento (POES) y su influencia en la inocuidad de los productos 
cárnicos en una empresa del rubro alimentario. Tesis para optar el título de: Ingeniero Industrial, Universidad Privada del Norte, Cajamarca, Perú. Recuperado de: https://repositorio.upn.edu.pe

Campoverde, P. (2015). Manual de Procedimientos, Buenas prácticas de Higiene, Manipulación y Seguridad Alimentaria para el área de Cocina del Hospital Moreno Vázquez Gualaceo. Ecuador, CUENCA. Recuperado de: https://dspace.ucuenca.edu.e c

Castañeda, R.; Fuentes, C.; Peñarrieta, J. (2016). Evaluación de los prerrequisitos del APPCC y análisis de los puntos críticos de control para el aseguramiento de la inocuidad en la producción de pan artesanal e industrial. Revista Boliviana de Química, vol. 33, 5, pp. 196-208. ISSN: 0250-5460. Recuperado de: https://www.redalyc.org/articu lo.oa?id=426349135007

Cunalata, Q., Gabriela, A. (2017). Diseño de un sistema de análisis de peligros y puntos críticos de control (APPCC) para el aseguramiento de la inocuidad de los productos elaborados en la industria de productos lácteos SOPRAB, (Tesis de grado) Universidad de Guayaquil, Facultad de
Ingeniería

Química,

Guayaquil,

Ecuador.

Recuperado

de

https://www.alimentosargenti nos.gob.ar/HomeAlimentos/

Díez, S. (2018). APPCC y prácticas higiénicas: estudio de caso en empresas de catering. Recuperado de https://www.corpoica.org.co/s itioweb/ Elearning/BPG

Garayoa, M., et al. (2010). Empresas de catering y seguridad alimentaria: Valoración del sistema APPCC. Recuperado de:

https://doi.org/10.1016/j.carbp ol.2010.02.012

Otegui, F., Michelini, E., Civit, D. (2017). Plan de análisis de peligros y puntos críticos de control (HACCP) para queso Gouda. Recuperado de https://www.fao.org.

Palomino, L. (2018). Guía de aseguramiento de la calidad sanitaria. S. d. s. d. I. E. Mexicanos. Recuperado de: https://www.anmat.gov.ar/Ali mentos/Enfermedades\%20tra nsmitidas\%20por\%20aliment os.pdf

Saltos, J., Márquez, Y., Demera, F., Alcívar,

B. (2019). Diagnóstico de la inocuidad del queso fresco en pequeñas empresas locales mediante el sistema HACCP. Revista Alimentos hoy, vol 27, 48. ISSN:

2027-291X. 
Recuperado

de:

https://alimentoshoy.acta.org.

co

Serrano, N. (2017). Inocuidad

alimentaria como aspecto clave en el sistema de gestion de calidad en elmer company. Universidad santo tomas, Bogotá, Colombia.

Recuperado de: http://repository.usta.edu.co

Vargas, S. (2016). Caracterización microbiologica de diversos tipos de queso elaborados en el Valle de Tulancingo Hidalgo. Departamento de Ciencias Agropecuarias, México, Universidad Autónoma del Estado de Hidalgo. Recuperado de: http://repository.uaeh.edu.mx 